\title{
Traumatisierungen bei Suchtpatienten - Relevanz und spezifische Behandlung in der ambulanten Suchttherapie
}

\author{
I. Schäfer \\ M. Schultz \\ U. Verthein \\ M. Krausz
}

\author{
Trauma in Addicted Patients - Relevance and Specific Interventions in \\ Outpatient Drug Services
}

\section{Zusammenfassung}

Bislang liegen kaum Informationen dazu vor, welche Bedeutung Traumatisierungen bei Patienten in der ambulanten Suchtbehandlung zugemessen wird. In einer bundesweiten Befragung in Einrichtungen der ambulanten Suchttherapie wurde deshalb erhoben, für wie relevant die dort tätigen Therapeuten Traumatisierungen halten und inwieweit sie im Rahmen der Therapie berücksichtigt werden. Aus 259 Einrichtungen konnten Angaben erhalten werden. Während nach Ansicht der teilnehmenden Personen Traumatisierungen bei durchschnittlich einem Drittel der Patienten eine wesentliche Rolle bei der Entstehung und/oder Aufrechterhaltung der Suchtproblematik spielen, wurden nur aus $18 \%$ der Einrichtungen spezifische Angebote berichtet. Häufig wurden Probleme in der Therapie Betroffener geschildert und der Wunsch nach spezifischer Weiterbildung geäußert. Die Ergebnisse unterstreichen die Notwendigkeit, auch ambulante Angebote weiter an die besonderen Bedürfnisse dieser Patientengruppe anzupassen.

\section{Abstract}

Information is scarce on how outpatient drug services in Germany see the importance of traumatic experiences in addicted patients. A German-wide investigation carried out in outpatient drug services explored the importance ascribed by therapists to traumatic experiences and to what extent these experiences are considered during treatment. Data were obtained from 259 institutions. According to the participants, traumatic experiences played a major part in the development of and/or persistence of addiction problems in one third of patients on average, but only $18 \%$ of the institutions offered specific treatment for these patients. The participants frequently described treatment problems and expressed the wish for specific further training. These results underline the necessity to further adapt also outpatient treatment offers to the special requirements of this group of patients.

Key words

Trauma $\cdot$ addiction $\cdot$ substance abuse $\cdot$ treatment

\section{Schliuisselwörter}

Trauma $\cdot$ Sucht $\cdot$ Substanzmissbrauch $\cdot$ Behandlung 
Die Lebensgeschichten suchtkranker Menschen sind häufig durch traumatische Erfahrungen geprägt. Zahlreiche Untersuchungen belegen, dass bei Personen in Suchtbehandlung hohe Raten interpersonaler Traumatisierungen im Kindesalter, wie sexueller Missbrauch, körperliche oder emotionale Misshandlung, anzutreffen sind $[1,2]$. Aber auch in späteren Lebensabschnitten sind Suchtpatienten weitaus häufiger als die Allgemeinbevölkerung traumatischen Erfahrungen ausgesetzt [3, 4]. Diese Erlebnisse und ihre Folgen wirken sich in erheblichem Maße auf den Verlauf der Abhängigkeitserkrankung und die Therapie aus. Oft sind die Betroffenen schlechter psychosozial integriert, weisen mehr Risikoverhalten und eine größere Anzahl von zusätzlichen Begleiterkrankungen auf [5 - 7]. Im Rahmen der Behandlung wurde bei traumatisierten Patienten eine schlechtere Therapieadhärenz mit geringeren Remissions- bzw. höheren Abbruchraten und häufigeren Rückfällen gefunden [6-9]. Insgesamt wird die Behandlung von traumatisierten Suchtpatienten von Therapeuten als deutlich schwieriger empfunden [10, 11]. Eine wichtige Bedeutung scheint dabei das breite Spektrum von psychischen Beeinträchtigungen zu besitzen, die in der Folge von Traumatisierungen auftreten können. Im Falle kindlicher Traumatisierungen zählen dazu unter anderem Ängstlichkeit, Aggression, Depressivität, Dissoziation, Symptome posttraumatischer Belastungsstörungen, Störungen des Selbstwertes, der Identität und des Bindungsverhaltens sowie Beeinträchtigungen der Fähigkeit zur Selbstregulation von Affekten und damit des Umgangs mit späteren stressvollen Lebensereignissen [12, 13]. Vor diesem Hintergrund wird der Zusammenhang zwischen traumatischen Lebenserfahrungen und substanzbezogenen Störungen häufig im Sinne der "Selbstmedikationshypothese“ verstanden [14]. Alkohol- oder Drogenkonsum beginnt demnach als ein teilweise erfolgversprechender Versuch, schmerzvolle oder in anderer Weise schwer erträgliche emotionale Zustände zu beeinflussen. Psychotrope Substanzen werden von den Betroffenen dabei nicht nur zur Dämpfung von negativen Affekten eingesetzt, sondern können bei eingeschränkter emotionaler Erlebnisfähigkeit auch positive Gefühle steigern oder weitere Traumafolgen wie Rückzugsverhalten und Störungen in der Gestaltung sozialer Kontakte positiv beeinflussen. Der Substanzkonsum kann so für die Opfer eine zentrale Funktion in der Sicherung elementarer Grundbedürfnisse einnehmen und als dysfunktionaler Versuch einer pharmakologischen Konfliktund Lebensbewältigung angesehen werden [15]. Aufgrund dieser Zusammenhänge kann Traumatisierungen und ihren Folgen eine entscheidende Bedeutung in der Behandlung betroffener Patienten zukommen und es erscheint unabdingbar, ihre besonderen Bedürfnisse im Rahmen der Therapie und bei der Gestaltung von Behandlungssettings zu berücksichtigen. In stationären Einrichtungen des Suchthilfesystems ist in den vergangenen Jahren eine wachsende Anzahl von spezialisierten Behandlungsangeboten für diese Patientengruppe entstanden. Über Indikationslisten und eine zunehmende Vernetzung ist es dabei besser möglich geworden, einen Eindruck von der inzwischen entstandenen Versorgungslandschaft zu erhalten. Demgegenüber liegen bislang kaum Informationen dazu vor, welche Bedeutung dem Problem von Traumatisierungen bei Suchtpatienten im ambulanten Teil des Suchthilfesystems zugemessen wird, in welchem Maße sie dort im Rahmen der Diagnostik berücksichtigt werden und ob auch hier bereits spezifische Versorgungsangebote etabliert wurden. Ziel der vorliegenden Untersuchung war es deshalb, einen Eindruck von der subjektiven Einschätzung der in der ambulanten Suchttherapie Tätigen bezüglich der Relevanz traumatischer Lebensereignisse bei ihren Patienten zu gewinnen. Weiter sollten Informationen dazu gewonnen werden, inwieweit diagnostische und therapeutische Maßnahmen in diesem Teil des Versorgungssystems auf traumatisierte Patienten abgestimmt sind.

\section{Material und Methoden}

\section{Stichprobe}

In einer postalischen Befragung wurden von den bundesweit etwa 2000 Einrichtungen des ambulanten Suchthilfesystems diejenigen um ihre Teilnahme gebeten, die im Jahr 2003 von mindestens einem Rehabilitationsträger als ambulante Therapieeinrichtung anerkannt waren. Eine entsprechende Zusammenstellung wird jährlich vom BKK-Bundesverband erstellt und beinhaltete in der zugrunde gelegten Fassung 467 Einrichtungen [16]. In einem Anschreiben wurde das Ziel der Untersuchung erklärt und um die Beantwortung eines für die Untersuchung zusammengestellten Fragebogens durch „eine/n therapeutisch tätige/n Mitarbeiter/in“ gebeten.

\section{Befragungsinstrument}

Um ein einheitliches Verständnis zu gewährleisten, enthielt die Einleitung des eingesetzten Fragebogens zunächst eine Traumadefinition, die sich an den „Traumakriterien“ der Posttraumatischen Belastungsstörung nach ICD-10 bzw. DSM-IV orientierte $[17,18]$. Der betreffende Abschnitt lautete: „Mit Traumatisierungen sind im Folgenden schwerwiegende, einschneidende Erlebnisse gemeint, die eine deutliche Bedrohung für die körperliche oder psychische Unversehrtheit des Betroffenen darstellen. Beispiele sind sexueller Missbrauch oder körperliche Misshandlung im Kindesalter bzw. Erfahrungen sexueller oder körperlicher Gewalt in späteren Lebensphasen. “ In einem allgemeinen Abschnitt wurden anschließend Daten zur ausfüllenden Person und zur Größe und Art der betreffenden Einrichtung erfasst. Neben Alter und Geschlecht wurde die Berufsgruppe der ausfüllenden Person erfragt, die Dauer der Erfahrung mit Suchtpatienten in Jahren und die Anzahl der aktuell betreuten Patienten. Die Fragen zur Einrichtung umfassten zunächst die jeweiligen Indikationsbereiche. Dabei wurden die Antwortalternativen der BKK-Indikationsliste vorgegeben (Alkohol-, Medikamenten-, Drogen-, Lösungsmittel- und Mehrfachabhängigkeit) mit der Möglichkeit von Mehrfachnennungen. Weiter wurde die Anzahl der in der Einrichtung durchschnittlich pro Monat betreuten Patienten erfragt sowie mögliche Einschränkungen für die Behandlung. Im Hauptteil des Fragebogens wurde zunächst eine Einschätzung dazu erbeten, bei welchem prozentualen Anteil der jeweiligen Patienten „Traumatisierungen eine wesentliche Rolle bei der Entstehung und/oder Aufrechterhaltung der Suchtproblematik spielen“. Weiter wurde darum gebeten, anhand vierstufiger Skalen die Notwendigkeit spezieller Angebote für diese Patientengruppe und spezieller Weiterbildungsmöglichkeiten für die Therapeuten zu beurteilen. Im letzten Abschnitt wurde erhoben, in welchem Umfang die befragten Einrichtungen auf die Betreuung traumatisierter Patienten ausgerichtet und in welcher Form 
traumaspezifische Angebote vorhanden waren. Weitere Fragen befassten sich mit dem Weiterbildungsstand des Personals und günstigen therapeutischen Rahmenbedingungen für die Behandlung traumatisierter Patienten.

\section{Auswertung}

Die Angaben zu allen Merkmalsbereichen wurden zunächst deskriptiv ausgewertet (statistische Kennwerte, Tabellen und Grafiken). Nach ihren Indikationsbereichen wurden die Einrichtungen unterschiedlichen Gruppen zugeteilt und auf Unterschiede im Hinblick auf die Anzahl behandelter Patienten untersucht. Zusammenhänge zwischen den Charakteristika der ausfüllenden Personen (Alter, Geschlecht, Berufsgruppe, Anzahl der monatlich behandelten Patienten, Berufserfahrung) bzw. der Einrichtungen (Einrichtungsgruppe, Behandlungsdauer) und der Einschätzung der Relevanz von Traumatisierungen wurden überprüft. Die Stärke der Zusammenhänge mit der Behandlungsdauer, dem Alter und der Berufserfahrung wurde mittels bivariater Korrelationen beschrieben, Gruppenunterschiede anhand der übrigen Variablen mittels t-test bzw. $\chi^{2}$-test auf Signifikanz getestet. Das Signifikanzniveau wurde auf $\alpha=0,05$ gesetzt. Zur Untersuchung von Interaktionseffekten zwischen Variablen, die Zusammenhänge zeigten, wurden mehrfaktorielle Varianzanalysen gerechnet.

\section{Ergebnisse}

Von den $\mathrm{n}=467$ Einrichtungen aus dem gesamten Bundesgebiet sandten $n=259$ den Fragebogen zurück (Rücklauf 56\%). In ca. 10 Einrichtungen war der Fragebogen kopiert und von mehreren Mitarbeitern ausgefüllt worden. In diesen Fällen wurde jeweils einer der Bögen nach dem Zufallsprinzip ausgewählt, um keine redundanten Informationen zu den betreffenden Einrichtungen zu erhalten.

\section{Charakteristika der ausfüllenden Personen}

Tab. 1 zeigt Geschlecht, Alters- und Berufsgruppen der ausfüllenden Personen. Bei einer sehr ausgeglichenen Geschlechterverteilung betrug der Altersmittelwert der Stichprobe $M=46,3$ Jahre $(S D=7,0$, range $26-62$ Jahre).

Tab. 1 Geschlecht, Alters- und Berufsgruppen

\begin{tabular}{lll}
\hline Variablen & Ausprägung & Häufigkeit \\
\hline Geschlecht & weiblich & $51 \%(n=130)$ \\
& männlich & $49 \%(n=125)$ \\
\hline Altersgruppen & unter 40 Jahre & $16 \%(n=41)$ \\
& 40 bis 50 Jahre & $50 \%(n=128)$ \\
& über 50 Jahre & $34 \%(n=87)$ \\
\hline Berufsgruppen & Psychologie & $42 \%(n=107)$ \\
& Sozialpädagogik & $40 \%(n=102)$ \\
& Sozialarbeit & $8 \%(n=21)$ \\
& Pädagogik & $6 \%(n=14)$ \\
& Medizin & $3 \%(n=7)$ \\
& Soziologie & $2 \%(n=6)$ \\
\hline
\end{tabular}

Entsprechend dem Altersmittelwert berichtete die Mehrzahl der ausfüllenden Personen über eine langjährige Erfahrung in der Arbeit mit Suchtpatienten $(M=14,2$ Jahre; $S D=7,2$; range $1-33$, $\mathrm{n}=257$ ). Eine mindestens 10 -jährige Erfahrung wiesen $71 \%$ der Stichprobe auf. Monatlich wurden im Mittel 28 Patienten persönlich betreut ( $S D=20,3$; range $2-140$ ).

\section{Charakteristika der Einrichtungen}

Die Einrichtungen ließen sich anhand der angegebenen Indikationsbereiche danach unterscheiden, ob sie ausschließlich alkoholabhängige und medikamentenabhängige Patienten betreuten, ob sie zusätzlich auch auf drogenabhängige bzw. polytoxikomane Patienten eingestellt waren oder ob ihr Angebot ausschließlich auf die letztgenannte Patientengruppe ausgelegt war (Tab. 2). Zusätzliche Indikationen wurden für ein Viertel der Einrichtungen berichtet $(n=65)$. Bis auf drei Einrichtungen betraf dies ausschließlich die beiden erstgenannten Gruppen von Einrichtungen und in über $80 \%$ der Fälle handelte es sich um die Indikationen „Essstörungen“ oder „Spielsucht“. Lediglich für drei Einrichtungen wurden zusätzliche Indikationen wie „psychische Störungen“ oder „psychiatrische Komorbidität“ genannt. Zugleich stellte eine psychiatrische Komorbidität die häufigste Einschränkung bezüglich einer Therapie dar. So wurde für 83 (32\%) Einrichtungen berichtet, dass eine Therapie komorbider Patienten nicht möglich sei. Weitere, seltener genannte Einschränkungen betrafen u.a. eine mangelnde Abstinenzfähigkeit $(n=8,3 \%)$ und geschlechtsspezifische Angebote $(n=10,4 \%)$. Zwischen den drei Einrichtungstypen zeigten sich keine signifikanten Unterschiede im Hinblick auf die Anzahl der pro Monat betreuten Patienten.

Tab. 2 Charakteristika der Einrichtungen

\begin{tabular}{|lll}
\hline Variablen & Ausprägung & Häufigkeit \\
\hline Einrichtungstyp & Alk & $46 \%(n=118)$ \\
& Alk/Drog & $37 \%(n=97)$ \\
& Drog & $17 \%(n=43)$ \\
\hline Anzahl der Patienten & $<50$ Pat./Monat & $19 \%(n=47)$ \\
& $50-100$ Pat./Monat & $35 \%(n=84)$ \\
& $101-200$ Pat./Monat & $37 \%(n=90)$ \\
& $>200$ Pat./Monat & $9 \%(n=23)$ \\
durchschnittliche & $<3$ Monate & $15 \%(n=35)$ \\
Behandlungsdauer & $3-6$ Monate & $33 \%(n=76)$ \\
& $7-12$ Monate & $38 \%(n=87)$ \\
& $>12$ Monate & $14 \%(n=32)$ \\
\hline
\end{tabular}

Anmerkungen: Alk: Hauptindikationen Alkohol-/Medikamentenabhängigkeit; Alk/Drog: zusätzlich Indikation Drogenabhängigkeit/Polytoxikomanie; Drog: Hauptindikation Drogenabhängigkeit

\section{Relevanz von Traumatisierungen}

Die subjektive Einschätzung der Therapeuten, bei welchem prozentualen Anteil der jeweiligen Patientinnen und Patienten „Traumatisierungen eine wesentliche Rolle in der Entstehung und/oder Aufrechterhaltung der Suchtproblematik spielen“, ergab über die gesamte Stichprobe hinweg einen Mittelwert von $33 \%$ der Patienten ( $S D=23,8$, range $0-100 \%, n=249$ ). Zwei Drittel (68\%) der teilnehmenden Personen schätzte diesen Anteil auf mindestens $20 \%$ aller Patienten. Während männliche Teilneh- 
mer eine bedeutsame Rolle von Traumatisierungen bei durchschnittlich 27\% (SD = 21,0) ihrer Patientinnen und Patienten vermuteten, lag dieser Wert bei weiblichen Teilnehmerinnen mit $39 \%(\mathrm{SD}=25,1)$ signifikant höher $(t=4,08, d f=236, p<0,001)$. Auch die durchschnittliche Behandlungsdauer zeigte einen, wenn auch schwachen, Zusammenhang mit dem Anteil der Patienten, bei denen Traumatisierungen als relevant eingeschätzt wurden $(r=.15, p=, 021)$. Anhand einer zweifaktoriellen Varianzanalyse wurde der Einfluss der Indikationsgruppen bestätigt, ein Interaktionseffekt zwischen dem Geschlecht und der Einrichtungsgruppe lag jedoch nicht vor. Bei der Überprüfung der Behandlungsdauer als Kovariate zeigte sich deren Einfluss auch im multivariaten Modell; auch hier blieb der Geschlechtsunterschied davon jedoch unbeeinflusst. Keine Zusammenhänge mit der Einschätzung der Relevanz zeigten die Zugehörigkeit zu den verschiedenen Berufsgruppen, die Anzahl der monatlich behandelten Patienten, das Alter sowie die Berufserfahrung. Wurden die Mittelwerte zwischen den drei Einrichtungstypen verglichen („Alk“, „Alk/Drog“, „Drog“), so zeigte sich, dass in den Einrichtungen, die ausschließlich Drogenpatienten behandelten, diese Zahl signifikant höher eingeschätzt wurde. Während sich in den ersten beiden Typen von Einrichtungen Mittelwerte von 27\% $(S D=21,0)$ bzw. $31 \%(S D=21,3)$ ergaben, wurde in diesen Einrichtungen geschätzt, dass Traumatisierungen bei durchschnittlich 50\% (SD = 50,1) der Patienten eine „wesentliche Rolle“ spielten $(\mathrm{p}<0,001)$.

Auf die offene Frage nach Besonderheiten im Therapieverlauf bei betroffenen Patienten wurde in $76 \%(n=199)$ der Fälle geantwortet. Am häufigsten wurde berichtet, dass es zu Problemen in der therapeutischen Beziehung komme („therapeutisches Bündnis schwer zu etablieren“, „Kontaktabbrüche“, „Übertragungsphänomene“, „Unsicherheit der Therapeuten“...), dass Schwierigkeiten im Verlauf der Therapie aufträten („können Grundvoraussetzung der Abstinenz nicht erfüllen“, „Verlängerung der Therapiedauer“, „,häufigere Rückfälle“, „lange Stabilisierungsphase“...) und dass bei Betroffenen in besonderem Maße komorbide psychische Störungen vorlägen („Ängste“, „Depression“, „Autoaggression“, „Suizidalität“, „Persönlichkeitsstörungen“....). Insgesamt wurde ein hoher Bedarf an spezifischen Angeboten für betroffene Patienten gesehen. 87\% $(n=223)$ der teilnehmenden Personen waren der Ansicht, dass solche Angebote „eher wichtig“ bzw. „wichtig“ seien (Abb.1).

In noch höherem Maße war dies für Weiterbildung und fachliche Unterstützung bezüglich traumaspezifischer Suchttherapie der Fall, die 95\% ( $n=243)$ für „wichtig“ bzw. „eher wichtig“ ansahen (Abb. 2).

\section{Aktueller Umgang mit Traumatisierungen}

Zur Diagnostik von Traumatisierungen berichteten 44\% ( $n=111)$, dass in ihrer Einrichtung systematisch traumatische Lebenserfahrungen exploriert würden. $16 \%(n=40)$ gaben an, systematisch eine Sexualanamnese zu erheben. Lediglich knapp 10\% der Gesamtstichprobe $(n=24)$ berichteten, dass traumatische Erlebnisse standardisiert anhand von speziellen Instrumenten oder Fragebögen erhoben würden.

Auf die Frage, ob traumaspezifische Angebote vorgehalten würden, berichteten $18 \%$ der ausfüllenden Personen $(n=46)$, dass

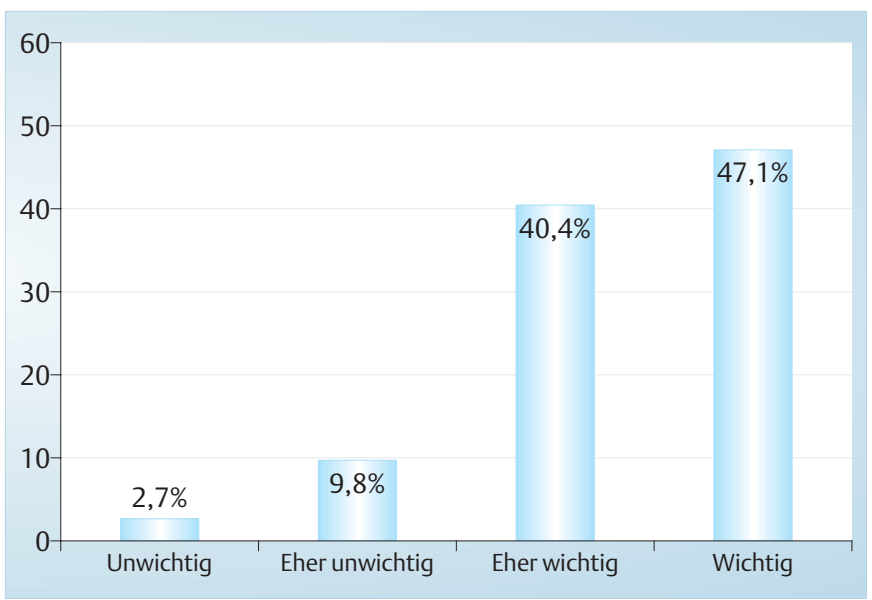

Abb. 1 „Wie wichtig sind Ihrer Meinung nach spezielle Angebote für Klientinnen und Klienten mit traumatischen Lebensereignissen?“

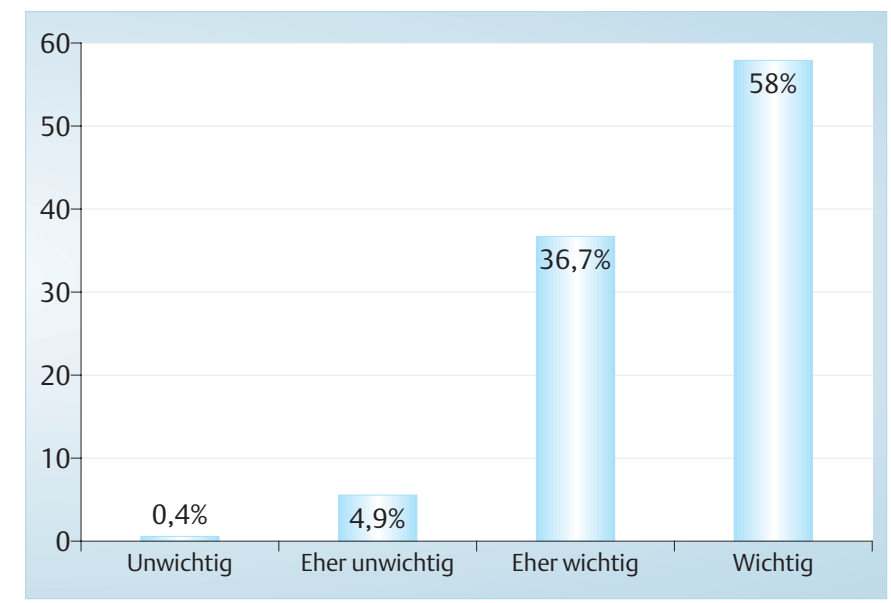

Abb. 2 „Wie wichtig wären nach Ihrer Einschätzung Weiterbildung bzw. fachliche Unterstützung bezüglich traumaspezifischer Suchttherapie?“

dies in ihrer Einrichtung der Fall sei. Am häufigsten wurde berichtet, dass dies „in der Einzeltherapie berücksichtigt“ werde $(n=14)$, dass Stabilisierungstechniken $(n=9)$, verhaltenstherapeutische Interventionen $(n=7)$ oder Eye Movement Desensitization and Reprocessing (EMDR) eingesetzt würden $(n=6)$ bzw. dass (Indikations-)Gruppen vorhanden seien $(n=6)$. Während die Mitarbeiter dieser Einrichtungen den Anteil von Patienten, bei denen Traumatisierungen eine bedeutsame Rolle spielen, auf durchschnittlich $44 \%$ ( $S D=25,1$ ) schätzten, betrug auch dort der Anteil der Personen, von denen angegeben wurde, dass sie an einer solchen Intervention teilnähmen, lediglich $17 \%(S D=23,4)$. Über die gesamte Stichprobe hinweg zeigte sich zwischen der Einschätzung der Relevanz von Traumatisierungen und dem Anteil der Patienten, die spezifische Interventionen erhalten, eine noch deutlichere Diskrepanz (Tab. 3).

$\mathrm{Zu}$ ihrem persönlichen Weiterbildungsstand berichteten 27\% ( $n=69)$ der ausfüllenden Personen, über eine „psychotherapeutische Zusatzqualifikation" zu verfügen. Im Bereich Traumatherapie berichteten $41 \%(n=106)$, bereits eine „(Kurz-)Fortbildung" besucht zu haben, jedoch nur 6\% $(\mathrm{n}=15)$ einen „Lehrgang“. 
Tab. 3 Subjektive Einschätzung der Bedeutung und Teilnahme an spezifischen Angeboten nach Einrichtungstypen

\begin{tabular}{lll}
\hline Einrichtungstyp & $\begin{array}{l}\text { Anteil der Patienten „bei } \\
\text { denen Traumatisierun- } \\
\text { gen eine wesentliche } \\
\text { Rolle ... spielen“ }\end{array}$ & $\begin{array}{l}\text { Anteil der Patienten } \\
\text { "die an einer (trau- } \\
\text { ma-)spezifischen Inter- } \\
\text { vention teilnehmen“ }\end{array}$ \\
\hline Alk & $\mathrm{M}=27,4 \%(\mathrm{SD}=21,0)$ & $\mathrm{M}=2,7 \%(\mathrm{SD}=6,8)$ \\
\hline Alk/Drog & $\mathrm{M}=31,3 \%(\mathrm{SD}=21,6)$ & $\mathrm{M}=3,7 \%(\mathrm{SD}=13,1)$ \\
\hline Drog & $\mathrm{M}=50,1 \%(\mathrm{SD}=27,7)$ & $\mathrm{M}=9,3 \%(\mathrm{SD}=20,8)$
\end{tabular}

Anmerkungen: „Alk“: Hauptindikationen Alkohol-/Medikamentenabhängigkeit ( $n=113)$; „Alk/Drog“: zusätzlich Indikation Drogenabhängigkeit/Polytoxikomanie ( $n=93)$; „Drog“: Hauptindikation Drogenabhängigkeit $(n=42)$

Auf die offene Frage nach wichtigen therapeutischen Rahmenbedingungen bei der Behandlung betroffener Patienten wurden von insgesamt $n=122$ Personen Angaben gemacht (47\%). Oft wurde darauf hingewiesen, dass traumatisierte Patienten besonders niedrigschwellige und flexible Angebote benötigten. Dies betreffe die Kriterien für eine Aufnahme in die Behandlung, etwa die oft zunächst nicht zu erreichende Abstinenz, aber auch eine möglichst geringe Wartezeit und eine geschlechtsspezifische Beratung und Therapeutenwahl. Besonders häufig wurde die Notwendigkeit hochfrequenter, langfristiger Therapieangebote mit hoher Beziehungskontinuität betont und die Schwierigkeit, entsprechende Zusagen von den Kostenträgern zu erhalten. So würden viele Patienten kontinuierliche Einzelgespräche in kurzen Intervallen benötigen und zusätzliche engmaschige Kontaktangebote, ggf. auch abends und an Wochenenden. Auch die Nachbetreuung müsse dem besonderen Bedarf der Patienten entsprechend kurzfristig intensiviert werden können. Ein weiterer besonders häufig geäußerter Aspekt betraf eine bessere Vernetzung mit spezialisierten niedergelassenen Kollegen, stationären und teilstationären Einrichtungen. Viele der ausfüllenden Personen beklagten einen Mangel an qualifizierten, regional erreichbaren Psychiatern und Psychotherapeuten, die ggf. konsiliarisch konsultiert werden könnten oder für die weitere Behandlung Betroffener zur Verfügung stünden. Auch die Möglichkeit, Patienten an spezialisierte Kliniken zu verweisen oder dort kurzfristig zur Krisenintervention aufnehmen zu lassen, sei bei weitem zu selten gegeben. Erneut wurde schließlich nachdrücklich der Wunsch nach qualifizierter Weiterbildung der Mitarbeiterinnen und Mitarbeiter zu traumaspezifischen Themen geäußert.

\section{Diskussion}

Ziel der vorliegenden Untersuchung war es, einen Eindruck davon zu erhalten, welcher Stellenwert Traumatisierungen bei Personen mit Abhängigkeitserkrankungen von den Mitarbeiterinnen und Mitarbeitern ambulanter Suchttherapieeinrichtungen zugemessen wird. Bereits die für postalische Befragungen vergleichsweise hohe Teilnehmerquote (56\%) deutete dabei darauf hin, dass an dieser Fragestellung ein erhebliches Interesse bestand. Explizit wurde dies in entsprechenden Kommentaren auf vielen der zurückgesandten Bögen geäußert. Nach Einschätzung der teilnehmenden Personen spielen Traumatisierungen bei einem erheblichen Anteil der Patientinnen und Patienten „eine wesentliche
Rolle bei der Entstehung und/oder Aufrechterhaltung der Suchtproblematik“. Er wurde über die gesamte Stichprobe hinweg auf ein Drittel geschätzt, annähernd 70\% der teilnehmenden Personen schätzten ihn auf mindestens ein Fünftel aller Patienten. In Einrichtungen für drogenabhängige bzw. polytoxikomane Patienten wurde dieser Anteil mit der Hälfte aller dort behandelten Personen angegeben. Selbst wenn in Betracht gezogen wird, dass sich an der Befragung in besonderem Maße Mitarbeiter beteiligten, die bereits für dieses Themenfeld sensibilisiert waren, unterstreicht dies die hohe therapeutische Relevanz von Traumatisierungen. So wurde von den Teilnehmern eine Vielzahl von Problemen bei der Behandlung traumatisierter Patienten berichtet, die sich mit den Befunden empirischer Studien decken [7-9]. Während Befunde zur Häufigkeit von Traumatisierungen und zu den besonderen Bedürfnissen traumatisierter Suchtpatienten bislang vorwiegend im angloamerikanischen Raum durchgeführt wurden, zeigt die vorliegende Untersuchung dabei für ein bedeutendes Segment des deutschen Suchthilfesystems, dass sich die hier Tätigen in identischer Weise mit diesen Problemen konfrontiert sehen. Diese Einschätzung war unabhängig vom Alter, der Zugehörigkeit zu einer bestimmten Berufsgruppe und der Berufserfahrung. Allerdings zeigten sich deutliche Geschlechtsunterschiede, die auch unter Kontrolle anderer Variablen bestehen blieben. Dieser Befund könnte darauf hinweisen, dass weibliche Therapeuten Traumatisierungen eher in Erwägung ziehen und häufiger entsprechende Gesprächsangebote machen. Andererseits wäre auch denkbar, dass Betroffene ihre Erfahrungen weiblichen Therapeuten eher mitteilen.

Von der überwiegenden Mehrzahl der teilnehmenden Personen wurde die Wichtigkeit spezifischer Angebote für die betroffenen Patienten unterstrichen. Dies stand in deutlicher Diskrepanz zur Anzahl der Einrichtungen, die bereits entsprechende Angebote implementiert hatten. Der Grund dafür scheint zum einen in den dafür notwendigen Rahmenbedingungen zu liegen. So sind viele Aspekte eines differenzierten Angebots, wie die Durchführung spezifischer Gruppen oder eine adäquate Supervision, nur bei einer besseren personellen und finanziellen Ausstattung möglich. Häufig sprengt auch die oft längere Therapiedauer bei betroffenen Patienten den von den Kostenträgern diktierten Rahmen. Andererseits sind spezialisierte Angebote auch nicht in jedem Setting sinnvoll zu integrieren und es stellt sich die Frage, ob jede Einrichtung „alles anbieten“ und jede therapeutische Person „alles können“ muss. Auch wenn für posttraumatische Störungen bei Suchtpatienten gezeigt werden konnte, dass deren integrative Behandlung durch dieselben Therapeuten mit höherer Wahrscheinlichkeit zu Therapieerfolgen führt [19-22] und auch von den Patienten bevorzugt wird [23], müssen alternative Modelle erwogen werden, die sich für viele Einrichtungen als passender erweisen könnten. Das Spektrum alternativer Möglichkeiten könnte sich dabei von der konsiliarischen Beratung durch Traumatherapeuten über die Durchführung spezialisierter Gruppenangebote durch externe Therapeuten bis hin zu einer sequenziellen Therapie erstrecken. In jedem Fall dürfte einer intensivierten Zusammenarbeit mit spezialisierten Angeboten bzw. Therapeuten sowohl innerhalb als auch außerhalb des Suchthilfesystems eine Schlüsselrolle zukommen. Ein wichtiges Ziel, das auch von vielen der Befragten geäußert wurde, wäre der Aufbau lokaler Netzwerke, die unter den jeweiligen Gegebenheiten eine optimale, an die individuellen Bedürfnisse der 
Betroffenen adaptierte Behandlung ermöglichen. Auch wenn dabei in manchen Fällen eine Überweisung die sinnvollste Alternative darstellen sollte, setzt dies eine adäquate Diagnostik in suchttherapeutischen Einrichtungen voraus. Aufgrund der Häufigkeit von Traumatisierungen bei Patienten mit Abhängigkeitserkrankungen sollte dies eine „Minimalanforderung“ an alle in der Suchttherapie Tätigen darstellen. In der vorliegenden Untersuchung berichteten lediglich $43 \%$ der Therapeutinnen und Therapeuten, dass traumatische Lebensereignisse in ihren Einrichtungen systematisch erfragt würden. Da damit gerechnet werden muss, dass Personen bzw. Einrichtungen mit Interesse an der Fragestellung in der Untersuchungsstichprobe eher überrepräsentiert waren, dürfte diese Zahl in der Gesamtheit der ambulanten Einrichtungen noch niedriger liegen. Besonders interpersonale Traumatisierungen, wie sexuelle und physische Gewalt, werden von Betroffenen jedoch weit eher berichtet, wenn sie von therapeutischen Personen systematisch erfragt werden (z. B. [24]). Häufig wird befürchtet, dass eine Exploration solcher Erlebnisse von den Klienten eher abgelehnt wird [25]. Wenn sie in ein Hilfsangebot eingebettet ist, wird sie von einem Großteil der Betroffenen jedoch als hilfreich empfunden [26, 27]. Einen wichtigen Beitrag dazu, die Diagnostik von Traumatisierungen und traumabezogenen Störungen weiter zu verbessern, kann dabei eine angemessene Weiterbildung leisten. In entsprechenden Fortbildungen könnte mit vertretbarem Aufwand zumindest ein Grundlagenwissen im Bereich der Psychotraumatologie und zu speziellen Aspekten bei Patienten mit Abhängigkeitserkrankungen erworben werden. Wie sich in der Untersuchung zeigte, hat allerdings nur ein geringer Anteil der Befragten bislang spezifische Lehrgänge durchlaufen und 95\% gaben an, dass sie eine weitere Qualifikation in diesem Bereich für wichtig hielten. Zusammenfassend kann gesagt werden, dass Traumatisierungen bei Suchtpatienten in Einrichtungen der ambulanten Suchttherapie, die heute den größten Anteil von Patienten behandeln, eine erhebliche Bedeutung zugesprochen wird. Ein Teil der Einrichtungen trägt dem auch bereits in Form spezialisierter Angebote Rechnung. Allerdings befindet sich diese Entwicklung, bezogen auf das Ausmaß des Problems, noch am Anfang. Zum einen sind künftig die Kostenträger gefordert, den nötigen finanziellen Rahmen für eine adäquate Therapie traumatisierter Patienten zu schaffen. Auf Seite der Einrichtungen sollte hingegen überprüft werden, in welchem Maße eigene Konzepte modifiziert und Behandlungsangebote ergänzt werden sollten und wie durch eine bessere lokale Vernetzung und umfassende Fortbildung dazu beigetragen werden kann, die vorhandenen Angebote weiter auf die Bedürfnisse dieser Patientengruppe abzustimmen.

\section{Literatur}

${ }^{1}$ Simpson TL, Miller WR. Concomitance between childhood sexual and physical abuse and substance use problems. Clinical Psychology Review 2002; $22: 27-77$

${ }^{2}$ Langeland W, Hartgers C. Child sexual and physical abuse and alcoholism: A review. Journal of Studies on Alcohol 1997; 59: 336-348

${ }^{3}$ Cottler LB, Nishith P, Compton WM et al. Gender differences in risk factors for trauma exposure and post-traumatic stress disorder among inner-city drug abusers in and out of treatment. Compr Psychiatry 2001; 42 (2): $111-117$
${ }^{4}$ Kilpatrick D, Acierno R, Resnick $\mathrm{H}$ et al. A 2-Year longitudinal analysis of the relationship between violent assault and substance use in women. Journal of Consulting and Clinical Psychology 1997; 65 (5): $834-847$

${ }^{5}$ Kang SY, Deren S, Goldstein MF. Relationships between childhood abuse and neglect experience and HIV risk behaviors among methadone treatment drop-outs. Child Abuse Negl 2002; 26: 1275-1289

${ }^{6}$ Ouimette P, Finney J. MR. Two-year posttreatment functioning and coping of substance abuse patients with posttraumatic stress disorder. Psychology of Addictive Behaviors 1999; 13: 105- 114

${ }^{7}$ Hien DA, Nunes E, Levin FR et al. Posttraumatic stress disorder and short-term outcome in early methadone treatment. Journal of Substance Abuse Treatment 2000; 19 (1): 31 - 37

${ }^{8}$ Najavits L, Weiss R, Shaw S. A clinical profile of women with posttraumatic stress disorder and substance dependence. Psychology of Addictive Behaviors 1999; 13: 98-104

${ }^{9}$ Ouimette PC, Moos RH, Finney JW. Two-year mental health service use and course of remission in patients with substance use and posttraumatic stress disorders. Journal of Studies on Alcohol 2000; 61 (2): $247-253$

${ }^{10}$ Brady KT, Killeen TK, Saladin ME et al. Comorbid substance abuse and posttraumatic stress disorder: Characteristics of women in treatment. American Journal on Addictions 1994; 3 (2): 160 - 164

${ }^{11}$ Najavits L. Clinicians' views on on treating posttraumatic stress disorder and substance use disorder. Journal of Substance Abuse Treatment 2002; 22: 79-85

12 Briere J. Treating adult survivors of severe childhood abuse and neglect: Further development of an integrative model. In: Jenny C (Hrsg). The APSAC handbook on child maltreatment. Newbury Park, CA: Sage Publications, 2002

${ }^{13}$ Herman J, Van der Kolk B. Traumatic antecedents of borderline personality disorder. In: Van der Kolk B (Hrsg). Psychological Trauma. Washington DC: American Psychiatric Press, 1987: 111-126

${ }^{14}$ Khantzian E. The self-medication hypothesis of addictive disorders: focus on heroine and cocaine dependence. American Journal of Psychiatry $1985 ; 142: 1259-1264$

${ }^{15}$ Krausz M, Schäfer I, Lucht M et al. Suchterkrankungen. In: Egle U, Hoffmann S, Joraschky P (Hrsg). Sexueller Mißbrauch, Mißhandlung, Vernachlässigung. Stuttgart, New York: Schattauer, 2004

${ }^{16}$ BKK Bundesverband. BKK Suchtinfo 2003. Essen, 2002

17 World Health Organization. International Classification of Diseases. Vol. 10th edition. Geneva: World Health Organization, 1992

${ }^{18}$ American Psychiatric Association. Diagnostic and statistical manual of mental disorders. Vol. Fourth edition. Washington DC: American Psychiatric Press, 1994

${ }^{19}$ Abueg FR, Fairbank JA. Behavioral Treatment of Posttraumatic Stress Disorder and Co-occurring Substance Abuse. In: Saigh PA (Hrsg). Posttraumatic Stress Disorder: A Behavioral Approach to Assessment and Treatment. Boston: Allyn and Bacon, 1992: 111 - 146

${ }^{20}$ Evans K, Sullivan J. Treating addicted survivors of trauma. New York: Guilford Press, 1995

${ }^{21}$ Najavits LM, Weiss RD, Liese BS. Group cognitive-behavioral therapy for women with PTSD and substance use disorder. Journal of Substance Abuse Treatment 1996; 13: $13-22$

22 Expert Consensus Guideline Series. Treatment of posttraumatic stress disorder: The Expert Consensus Panels for PTSD. Journal of Clinical Psychiatry 1999; 60 (Suppl. 16): 3 - 76

${ }^{23}$ Brown P, Stout R, Gannon-Rowley J. Substance use disorder-PTSD comorbidity: Addiction and psychiatric treatment rates. Psychology of Addictive Behaviors 1998; 13: 115-122

${ }^{24}$ Simpson TL, Westerberg VS, Little LM et al. Screening for childhood physical and sexual abuse among outpatient substance abusers. J Subst Abuse Treat 1994; 11 (4): 347 - 358

${ }^{25}$ Salyers MP, Evans LJ, Bond GR et al. Barriers to Assessment and Treatment of Posttraumatic Stress Disorder and Other Trauma-Related Problems in People with Severe Mental Illness: Clinician Perspectives. Community Mental Health Journal 2004; 40 (1): $17-31$

${ }^{26}$ Newman E, Walker E, Gefland A. Assessing the ethical costs and benefits of trauma-focused research. General Hospital Psychiatry 1999; 21: $187-196$

${ }^{27}$ Griffin MG, Resick PA, Waldrop AE et al. Participation in Trauma Research: Is There Evidence of Harm? Journal of Traumatic Stress 2003; 16 (3): $221-227$ 